\title{
A new threshold-based traffic characterization and policing mechanism for communication networks
}

\author{
S. Durinovic-Johri \\ P. K. Johri
}

$A T \& T L a b s$

200 Laurel Avenue, Middletown, NJ 07748, U.S.A.

email:johri@att.com

\begin{abstract}
This paper develops a new threshold-based traffic characterization which has immense flexibility together with the ability to be very specific. It considerably generalizes the moving window and peak rate allocation schemes. A policing mechanism based on this characterization has a simpler implementation than the Leaky Bucket and can offer advanced services. The characterization is based on traffic measurements and an easy-to-implement procedure is developed to obtain parameters directly from user data. The characterization can be used in a "cold start" mode and quickly adapted as more data becomes available.
\end{abstract}

\section{INTRODUCTION}

The Asynchronous Transfer Mode (ATM) technology is being developed to support multi-service high-speed communication networks. The stringent performance desired of networks, such as the ATM network, requires that user traffic be characterized for the purposes of 
(a) allocating network resources,

(b) call admission control,

(c) usage parameter control or policing of user traffic, and

(d) charging.

Currently, user traffic is specified in statistical terms such as the peak rate, the average rate, and perhaps the largest burst size allowed (see, for example, Rathgeb (1993), Tutofor (1990)). Deterministic rule based traffic descriptors are used for monitoring and policing (Doshi (1994)). Many authors have noted the inadequacy of some of these characterizations (Butto (1991), Rathgeb (1992, 1993), Tutofor (1990)). In particular, it is common knowledge that the average rate cannot be measured or policed properly (Rathgeb (1992), Tutufor (1990)). Only the peak rate lends itself to easy monitoring and allocation of network resources. However, the network capacity is underutilized if a peak rate based characterization is used. The higher the peak rate compared to the average rate, the more severely the networks resources are underutilized.

The Leaky Bucket (Turner (1986)) is a device widely used for policing. It has two main parameters - the token pool size and the drain rate - and this limits the type of control that can be exercised over user traffic. It also limits the user's ability to accurately describe its traffic. Uncertainty at call setup and infrequent but large traffic spikes (Rathgeb (1992, 1993), Tutufor (1990)) make it difficult to properly dimension the Leaky Bucket. The Leaky Bucket parameters must be chosen conservatively to guard against excessive cell losses. Commonly assumed user traffic patterns do not pass transparently through a Leaky Bucket. For example, the exponentially distributed ON-OFF pattern (see, for example, Choudhury (1996)) and the more general $N$-state Markov chain (see, for example, Elwalid (1993)), go through a reasonably sized Leaky Bucket with large cell losses (Johri (1995)) relative to the desired network cell loss rate of $\approx 10^{-9}$. Further, widely differing user traffic patterns can be compliant with the same Leaky Bucket. The network will have to provide enough capacity for the "worst case" pattern coming out of the Leaky Bucket. This is, in some cases, but not always (Doshi (1994)), a deterministic ON-OFF traffic pattern and is again a conservative assumption.

Other commonly known policing mechanisms include the jumping window and moving window schemes (see, for example, Rathgeb (1993)). It is shown in Rathgeb (1993) that, from a performance perspective, the Leaky Bucket is superior to both these schemes.

The previous discussion points to a strong need for a new policing mechanism to overcome the shortcomings of the Leaky Bucket. The main requirement is to more closely relate allowable traffic patterns to the parameters of the policing mechanism. This requires a new way of characterizing user traffic. In addition, a 
simple mechanism is required for adapting the policing mechanism if it is too strict or too lenient. In this paper, we focus on measurability, adaptability, and flexibility, to develop a new threshold-based characterization scheme (TCS). This is a pattern based characterization and will permit more precise specification of allowable user traffic patterns. We also develop an easily implementable policing mechanism based on TCS. It is important to note that we develop TCS in its most general form. Simple versions of TCS, with just a few thresholds, will suffice for single users. TCS will use more parameters for services linking networks or involving a large amount of traffic.

This paper is organized as follows: In $\$ 2$ we highlight the need for policing. In $\S 3$ we show a typical user data stream which forms the basis of TCS. In $\$ 4$ we develop TCS and discuss its properties. In $\$ 5$ we develop a new policing mechanism based on TCS, provide a simple procedure for measuring traffic descriptors from user data, and address issues related to overdimensioning, sensitivity, and characterization under uncertainty. Finally, §6 contains the conclusions.

\section{THE NEED FOR POLICING}

Accurately characterizing user traffic is not an easy task. This is because the network will carry traffic from multiple users as well as other networks and may itself offer services, such as video services, which support user applications for which the traffic and demand is unclear. One common view is that the network (or the user) may never know enough about the user's applications to adequately characterize the user's traffic. Moreover, with high bandwidth more readily available, the network can afford to offer loosely policed and, consequently, inefficient services for carrying such traffic. The INTERNET provides this type of service. There is a per site access charge with users allowed to transmit as much as they like.

Without adequate policing, the network opens itself to exploitation. Suppose two users subscribe to a service at a rate of X dollars per month (see Figure 1a). This service is not adequately policed allowing the users to send as much traffic as they like.

A third entrepreneur can subscribe to the same service (see Figure 1b). This third user can now offer to transmit the previous two users' traffic at a reduced rate of $0.75 \mathrm{X}$ dollars per month, by multiplexing their traffic and sending it over its access line. As far as the original users are concerned, their traffic is going over the same network with the same performance guarantees as before but at $25 \%$ lower cost. The network, however, has lost $50 \%$ of its revenue while still carrying exactly the 
same traffic. The third user may need to provide some buffering or may have to subscribe to a slightly higher rate service to pull this through, but the profit would still be worth it.

Figure 1a: "Naive" users with loosely policed services

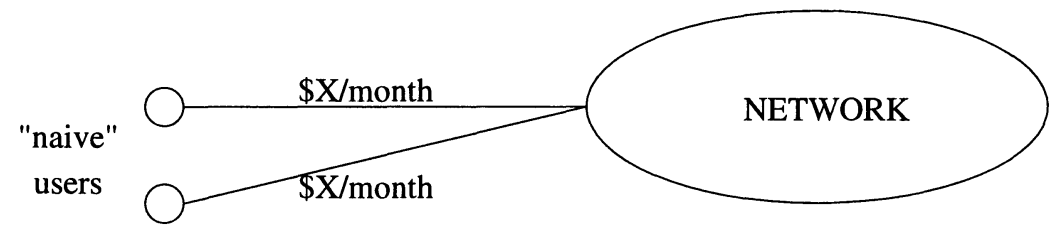

Figure 1b: "Smart" user reselling access

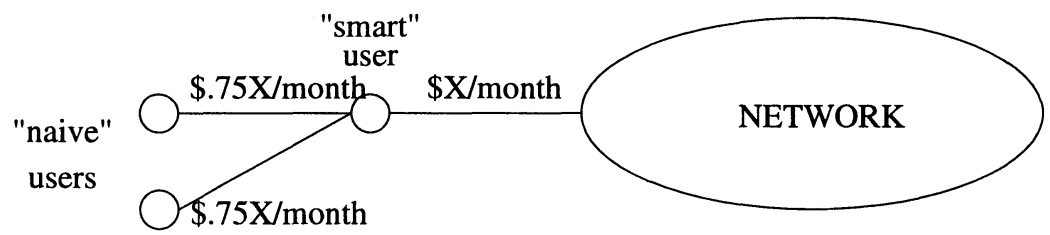

This example is not far fetched. The companies reselling INTERNET access are a prime example of such entrepreneurs. The only way to prevent this is to not allow the same traffic permitted by two $X$ dollars per month services on a service which costs substantially less than $2 \mathrm{X}$ dollars per month. In other words, to effectively police user traffic.

A contract is generally established between the user and the network specifying the traffic descriptors, the policing mechanism and the performance guarantees. The network may have no additional information about user traffic and, thus, has to infer its characteristics from the traffic descriptors specified in the contract. Consider two services with identical Quality of Service guarantees. The traffic descriptors in the first service allow a wide variety of traffic patterns while they are more limiting in the second. The network will have to devote more resources (buffers and/or bandwidth) to the first service than to the second, so that quality is assured even with the "worst case" user traffic pattern. Consequently, if services 
are priced according to the amount of network resources used, the first service will be more expensive.

The more precisely the network can specify the allowable user traffic patterns from the traffic descriptors, the more efficiently it can allocate its resources. This leads to lower operating cost but does not solve the problem that it is difficult to precisely characterize user traffic. However, it is important to realize that the latter is the user's responsibility. The network only needs to offer a variety of services, with lower prices for more precise specification of traffic descriptors. The user now has the option, for a given service quality, to choose vague traffic descriptors and pay higher prices, or choose precise traffic descriptors and pay lower prices.

\section{A SAMPLE USER TRAFFIC STREAM}

Several real examples of encoded video traffic were considered in Rathgeb (1992). One data set comprised of 171,000 frames generated at the rate of 24 frames per second. It was seen that the number of bytes per frame was highly variable and that there were numerous spikes in the data. The number of bytes produced by the encoder output was also recorded in fixed time intervals. For example, each frame could be "sliced" into 30 fixed time intervals. The burstiness in the slices was greater than in the frames.

For our purposes, it is sufficient to consider that a user produces a stream of cells (or packets, or frames, etc.) over time and that it is possible to measure and control the number of cells in fixed time intervals of length $t$. The value of $t$ can be negotiated between the network and the user and can be larger than or equal to the smallest time interval over which measurements are available. The traffic pattern, for example, may look like the one shown in Figure 2. This data is hypothetical and not taken from Rathgeb (1992). Figure 2 shows that 20 cells arrived in the first time interval, 30 cells arrived in the second time interval, and so on. Let $N$ denote the number of fixed time intervals for which user data is available, and let $c_{i}$ denote the number of cells in interval $i, i=1,2, \ldots, N$. Thus, in the Figure $2, N=100$ and $c_{1}=20, c_{2}=30$, and so on. The entire data stream is denoted as the sequence $\left\{c_{i}\right\}_{i=1}^{N}$. The maximum number of cells that can be produced in any time interval depends on the access line rate (or a negotiated peak rate) and is, say, 100 for the data in Figure 2. This traffic pattern will be used for illustrative purposes only. 
Figure 2: A Typical User Data Stream Displayed

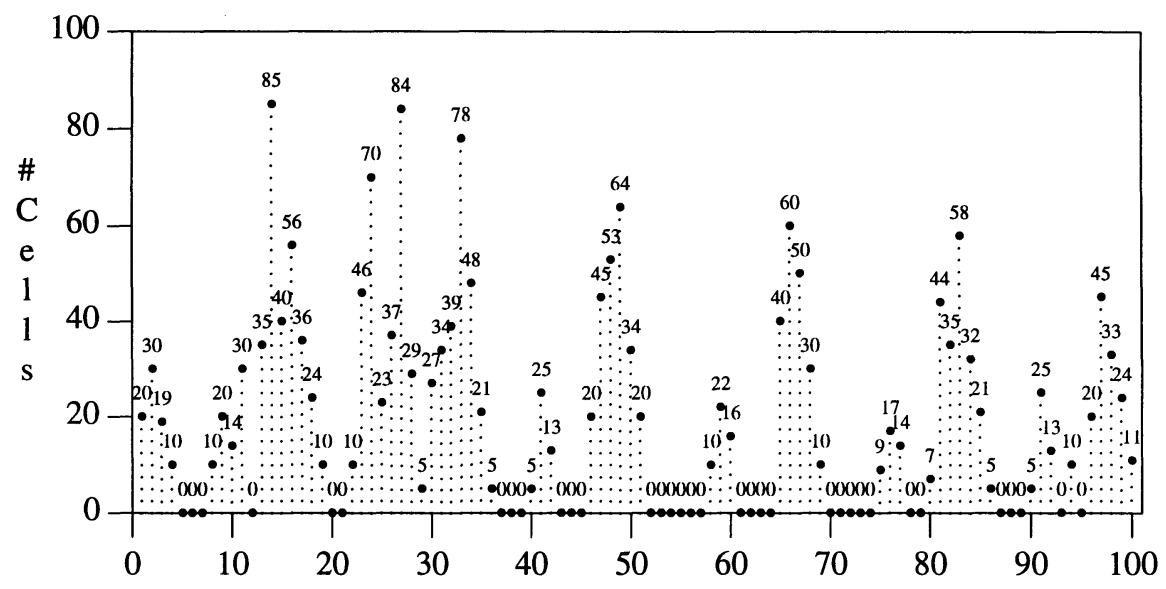

Time Intervals

\section{A THRESHOLD-BASED CHARACTERIZATION SCHEME}

This section develops the threshold-based characterization scheme (TCS). TCS tries to capture the heights and frequency of occurrence of the various peaks in the data.

TCS characterizes (and polices) traffic over a moving window of $n$ time intervals, where $n$ is a positive integer. The value of $n$ is negotiated between the user and the network and can differ from user to user.

For example, $n$ could be chosen as 10 . Now, the first moving window consists of time intervals $1,2, \ldots, 10$, the second of time intervals $2,3, \ldots, 11$ and so on.

The next step is to define the heights of the peaks. Again, to keep TCS very flexible, we allow a set of $m$ thresholds, where $m$ is a nonnegative integer. The value of $m$ and the value of each individual threshold is also negotiated between the user and the network and can vary from user to user. Alternatively, the network can offer several ready-made solutions for widely used applications. Let 
$\mathbf{h}=\left(h_{1}, h_{2}, \ldots, h_{m}\right)$ denote the values (in number of cells) of the $m$ thresholds chosen. Further, threshold $h_{m+1}$ is always taken to correspond to the access line or peak rate, that is, it represents the maximum number of cells that can arrive in any time interval. The thresholds $h_{j}$ must be increasing in $j$, that is, $0 \leq h_{1}<h_{2}<\cdots<h_{m}<h_{m+1}$.

For example, $m$ could equal 5 and a set of relevant thresholds can be $\mathbf{h}=(0,20,40,60,80)$. In addition, $h_{6}=100$.

The main idea in policing is to place limits on how much traffic is allowed in. This is done in TCS is terms of the maximum (number of) violations of each threshold allowed in the moving window of $n$ time intervals. Here a violation implies that the threshold is exceeded. Let $\mathbf{v}=\left(v_{1}, v_{2}, \ldots, v_{m}\right)$ denote the vector of maximum violations of thresholds $\mathbf{h}=\left(h_{1}, h_{2}, \ldots, h_{m}\right)$, respectively, allowed by TCS. By definition, threshold $h_{m+1}$ is violated 0 times. We have assumed that the peak rate is either limited by the access line rate or is being policed separately. Since the thresholds $h_{j}$ are increasing in $j$, the maximum violations $v_{j}$ must be nonincreasing in $j$ to be valid.

For example, we can specify $\mathbf{v}=(10,8,6,4,2)$ to impose the requirement that in any 10 consecutive times intervals, threshold 80 will not be exceeded more than 2 times, threshold 60 will not be exceeded more than 4 times, and so on. An easily implementable procedure is devised to measure these numbers from user data in §5.1. For the data in Figure 2, the threshold 80 is violated a maximum of 1 time (in any window which includes the interval 14 or the interval 27) and threshold 60 is violated a maximum of 3 times (in windows which include intervals $24,27, \& 33$ ).

Threshold Characterization Scheme : TCS consists of the time interval duration $t$, the window size $n$, the number of thresholds $m$, the threshold values $\mathbf{h}$ and $h_{m+1}$, and the maximum violations $\mathbf{v}$.

\subsection{Advantages of TCS}

The most important advantage is that TCS is based on user data streams. All parameters can be directly measured as well as observed visually. This allows a seamless transition from data measurements to policing. The measurements show how the data is varying and the policing is based on limits placed on the amount of variation allowed. The fact that both measurements and limits are in terms of the same quantities makes TCS very intuitive and easy to understand. This is not true for the second Leaky Bucket parameter - the token pool size. It is supposed to 
represent the maximum burst size allowable, but whether an actual burst of this size will be allowed in or not depends on the token pool state at the time of the start of the burst. Consider a Leaky Bucket with a drain rate of $1 \mathrm{cell} / \mathrm{msec}$ and a token pool size of 100 . A burst of 100 cells at the peak rate of 2 cells $/ \mathrm{msec}$ will be transmitted over 50 msecs. Consequently, cells will be lost unless the token pool has at least 50 tokens at the start of transmission, since only 50 tokens will be generated during transmission of the burst. This is highly unpredictable and is the factor which makes this Leaky Bucket parameter undesirable.

Another important advantage of TCS is that it is very flexible, in that any number and values of thresholds can be chosen, and yet can be made as precise as desired. This is, of course, at the expense of having a larger number of parameters. The Leaky Bucket, on the other hand, cannot be made precise.

As transmission speeds continue to increase, the time to transmit a cell becomes smaller and smaller. Policing can either be done cell by cell, as with the Leaky Bucket, or over small time intervals by limiting the number of cells allowed in these intervals. In both cases, it is imperative that the policing mechanism be very simple to implement and quick to run. This is, in fact, the most attractive feature of the Leaky Bucket and it is natural to expect that a policing mechanism based on TCS will be much more complex. In section 5, we will first develop a policing mechanism and show, contrary to expectation, that it is indeed quite simple.

\subsection{Generalizations of TCS}

The maximum violations in TCS control the number of times peaks can occur in any window. A user can still bunch the peaks together within a window. Additional traffic descriptors can be introduced to control the spacing between the peaks. In a generalized TCS, we do this by specifying the minimum number of time intervals which must separate two successive violations of the same threshold.

For example, we can impose the requirement that any two successive violations of threshold 80 must have at least 10 time intervals separating them, and so on.

A variation of TCS can be developed using the jumping window scheme. The full development of these generalizations is deferred for the future.

\subsection{Generality of TCS}

TCS can be used to police the peak rate. Let $x$ be the number of cells corresponding to the peak rate over a time interval. Then, threshold $h_{m}$ can be set to $x$ with $v_{m}=0$. This will prevent more than $x$ cells being transmitted in any 
time interval. Note that this is not identical to policing the peak rate with a Leaky Bucket type mechanism.

With $n=1$ and $m=1$, TCS reduces to the Jumping Window (Rathgeb (1993)) scheme with a window of duration $t$. TCS also generalizes the Moving Window scheme (Rathgeb (1993)) by allowing much finer granularity of control within each window defined by the various thresholds instead of a single number dictation the maximum allowance in each window.

\section{BASIC TCS-BASED POLICING MECHANISM}

This section describes a policing mechanism based on TCS. The following variables are used:

$B$ is an $m \times(n-1)$ matrix of bits, used to monitor violations of the $m$ thresholds over the last $n-1$ time intervals with $B_{i j}=1$ if threshold $h_{i}$ is violated in the $j$-th previous time interval and 0 otherwise.

$\mathbf{u}$ is a vector of $m$ integer variables used to monitor the total number of violations of threshold $\mathbf{h}$ in the last $n-1$ time intervals. Thus, $u_{i}=$ sum of elements in row $i$ of matrix $B$.

The variable $\mathbf{u}$ is not necessary and the same information can be derived from $B$.

The basic policing scheme is as shown in Figure 3. The policing mechanism cycles through steps 1-3. An alternate step 4 can be included to precalculate certain information which allows this procedure to run even faster. Step 2 is where all the policing is done. Note that this is the simplest step in the whole procedure and even simpler than the corresponding policing step in the Leaky Bucket mechanism. The other steps simply update the variables of the policing mechanism. Step 4 is performed in parallel with step 2 . 
Figure 3: Policing Mechanism without and with Precalculations

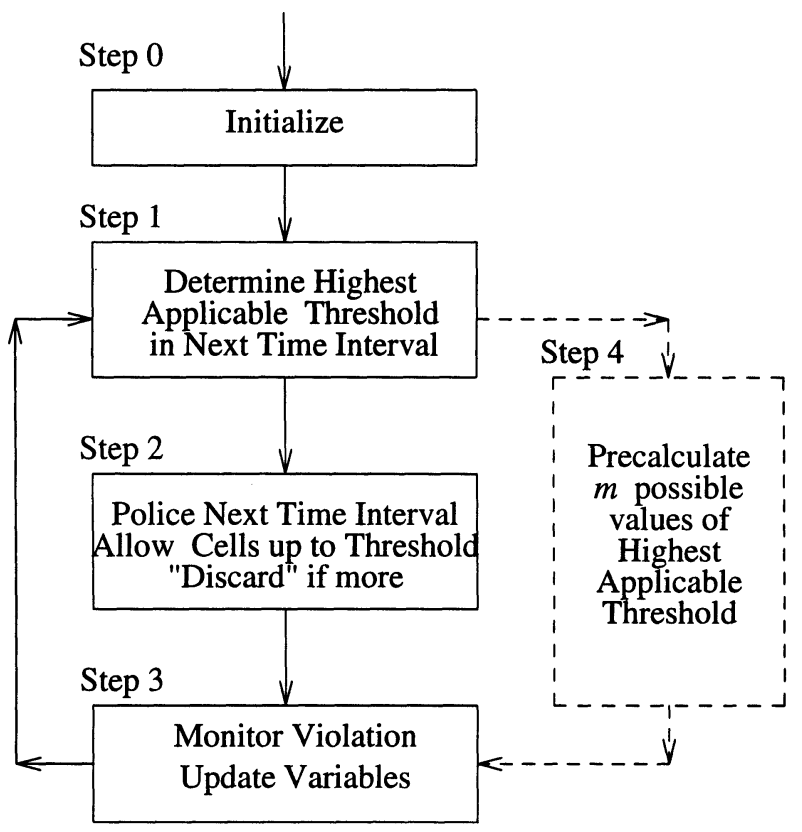

We now illustrate how the steps $0,1,3$ and 4 are performed. Assume the following TCS parameters: $m=5, \quad n=10, \quad \mathbf{h}=(0,20,40,60,80), \quad h_{6}=100$, and $\mathbf{v}=(8,6,3,1,1)$.

Step 0: Initialize Variables

Set $B_{i j}=0$, and $u_{i}=0$ for $i=1,2, \ldots, m$ and $j=1,2, \ldots, n-1$.

Assume that the current values of the variables are given below. These values are not based on the data in Figure 2.

$B=\left[\begin{array}{l}101101101 \\ 001101100 \\ 000100100 \\ 000100000 \\ 000000000\end{array}\right] \quad \mathbf{u}=(6,4,2,1,0)$ 
Let the current time interval be 10 . The value of $B$ indicates that threshold $5(=80)$ was not violated in time intervals $1-9$, threshold $4(=60)$ was violated only in time interval 4 , and so on. The value of $\mathbf{u}$ indicates that threshold $1(=0)$ was violated a total of 6 times in time intervals 1-9, threshold $2(=20) 4$ times, and so on.

\section{Step 1: $\quad$ Determine Highest Applicable Threshold in Next Time Interval}

In a "C"-like language, this is easily accomplished as follows:

$$
\begin{aligned}
& k=1 ; \\
& \text { While }\left(u_{k}<v_{k} \& k \leq m\right)++k ;
\end{aligned}
$$

The resulting value of $k(=\hat{k})$ yields the highest applicable threshold $h_{\hat{k}}$.

For the variable values given earlier, $\hat{k}=4$ and threshold $h_{4}(=60)$ is the highest applicable threshold in the next time interval.

\section{Step 3: $\quad$ Monitor Violation and/or Update Variables}

Let threshold $d(<\hat{k})$ be the highest threshold violated.

The value of $d$ depends on the number of cells transmitted in the last interval.

Update the variables as follows:

$B$ Delete column $n-1$.

Shift other columns to the right.

Set $B_{i 1}=1$ if $i \leq d$ and 0 otherwise

$\mathbf{u}$

row $\operatorname{sum} B$

Assume that $35\left(\leq h_{\hat{k}}=60\right)$ cells were transmitted in the last time interval. Then, $d=2$, and threshold $h_{2}(=20)$ is the highest threshold violated. The new values of the variables are given below.

$$
B=\left[\begin{array}{l}
110110110 \\
100110110 \\
000010010 \\
000010000 \\
000000000
\end{array}\right] \quad \mathbf{u}=(6,5,2,1,0)
$$

After each time interval, steps 3 and 1, in that order, yield the highest threshold applicable for the following time interval. The highest applicable threshold with the new variable values is again $h_{4}$.

\section{Step 4: $\quad$ Precalculate All Possible Values of Highest Applicable Threshold}

There are $\hat{k}-1(=3)$ possible values of $d$ the next time Step 3 is evaluated. It is easy to precalculate the highest applicable thresholds (repeat calculations in Step 3) for each of these possibilities. These are $\hat{k}=4,2$ and 2 , corresponding to $d=1,2$ 
and 3, respectively. With precalculations, steps 3 and 1 become very simple. Step 4 is the only complicated step. However it is done in parallel with step 2, and the whole duration $(t)$ of the time interval is available for performing step 4 .

The actual policing in Step 2 is done with just a counter, since cells are allowed in up to $h_{\hat{k}}$ cells and then discarded (or marked and let in). The counter mechanism leads to a simpler implementation than the counter and timer required for the Leaky Bucket. The full duration $(t)$ of the time interval is available for step 4 . Hence, the number of thresholds etc. has little impact on complexity. Moreover, advanced features can be built on this basic TCS based policing mechanism (see §5.7).

\subsection{Measuring Threshold Violations}

Let $\nabla_{y}(x)$ denote the indicator function of the event $(x>y)$, that is, $\nabla_{y}(x)$ equals 1 if $x>y$ and 0 otherwise. Then, $\nabla_{h_{j}}\left(c_{i}\right)$ indicates if the $j$-th threshold is violated in the $i$-th time interval, $j=1,2, \ldots, m, i=1,2, \ldots, N$. The sequence $\left\{\nabla_{h_{j}}\left(c_{i}\right)\right\}_{i=1}^{N}$ is a sequence of 0 's and 1's, that is, a bit stream. A simple program, perhaps implemented in hardware, can produce these $m$ bit streams.

The observed violations $\hat{v}_{j}$ of threshold $h_{j}$ can be calculated with the following two steps:

Step a: count $=\hat{v}_{j}=\sum_{i=1}^{n} \nabla_{h_{j}}\left(c_{i}\right)$;

Step b: For $(i=n+1 ; i \leq N ;++i)$

$$
\begin{aligned}
& \text { count }=\text { count }+\nabla_{h_{j}}\left(c_{i}\right)-\nabla_{h_{j}}\left(c_{i-n}\right) ; \\
& \text { If }\left(\hat{v}_{j}<\text { count }\right) \text { then } \hat{v}_{j}=\text { count }
\end{aligned}
$$

For the data in Figure 2, we obtain $\hat{\mathbf{v}}=(10,9,3,3,1)$.

\subsection{Maximum Allowance}

The peak rate based allocation can be very wasteful. A total of 1,940 cells are sent in by the user in the 100 intervals in Figure 2. If the user was allowed a peak rate of 100 cells in each time interval, the user could have sent a maximum of 10,000 cells. Hence, less than $20 \%$ of the peak allowance is actually used and more than $80 \%$ of the capacity is wasted with a peak rate based allowance.

TCS is a subpeak based allocation and, hence, should lead to reduced overdimensioning of the network. The maximum number of cells allowed by $\mathbf{v}$ over a window of size $n$ is given by 
$w(\mathbf{v})=v_{m} h_{m+1}+h_{1}\left(n-v_{1}\right)+\sum_{j=2}^{m} h_{j}\left(v_{j-1}-v_{j}\right)$

since the user can send $h_{m+1}$ cells no more than $v_{m}$ times, $h_{m}$ cells no more $v_{m-1}-v_{m}$ times, and so on. With $\mathbf{v}=(10,9,3,3,1)$, the user can send in no more than $w(\mathbf{v})=520$ cells in 10 time intervals. This allocated capacity is $52 \%$ of the capacity allocated with a peak rate based allocation. Dividing $w(\mathbf{v})$ by $n$ yields the maximum allowance on a per time interval basis.

\subsection{Sensitivity to Tunable Parameters}

As mentioned earlier, the parameters $t, n$, and $m$, and thresholds $\mathbf{h}, h_{m+1}$, can be tailored by the network to suit each user's needs. In Table 1 , we have calculated $\hat{\mathbf{v}}$ for various values of these parameters for the data in Figure 2. Next, we calculated $w(\mathbf{v}) / n$ assuming $\mathbf{v}=\hat{\mathbf{v}}$. The smaller the value of $w(\hat{\mathbf{v}}) / n$, the smaller the overdimensioning. Recall that $w(\mathbf{v}) / n=100$ corresponds to the peak rate. As can be seen from Table 1, larger values of $m$ and $n$ lead to smaller overdimensioning and a characterization which is closer to the actual user data distribution.

Table 1: Various TCS Characterizations of the Data in Figure 2

\begin{tabular}{|c|c|c|c|c|}
\hline$m$ & $\mathbf{h}$ & $n$ & $\hat{\mathbf{v}}$ & $w(\hat{\mathbf{v}}) / n$ \\
\hline 5 & $(0,20,40,60,80)$ & 5 & $(5,5,3,2,1)$ & 64 \\
5 & $(0,20,40,60,80)$ & 10 & $(10,9,3,3,1)$ & 52 \\
5 & $(0,20,40,60,80)$ & 20 & $(18,15,6,4,2)$ & 45 \\
10 & $(0,10,20, \ldots, 70,80,90)$ & 5 & $(5,5,5,5,3,2,2,1,1,0)$ & 58 \\
10 & $(0,10,20, \ldots, 70,80,90)$ & 10 & $(10,9,9,6,3,3,3,2,1,0)$ & 46 \\
10 & $(0,10,20, \ldots, 70,80,90)$ & 20 & $(18,15,15,11,6,5,4,3,2,0)$ & 40 \\
\hline
\end{tabular}

\subsection{Adapting TCS in Continuous Mode Operation}

Since it is very easy to observe the actual number of threshold violations $\hat{\mathbf{v}}$, it is also easy to continuously adapt TCS to achieve a desired level of cell losses. If there are too many (few) cell losses then the value of $\mathbf{v}$ can be increased (decreased) incrementally. At all times it is necessary to maintain

$\mathbf{v} \geq \hat{\mathbf{v}}$ 


\subsection{Characterization Under Uncertainty - Building in Slack}

To account for uncertainty in user behavior or insufficient user data, a slack or safety net of any desired size can be built into TCS. For example, we may have measured $\hat{\mathbf{v}}=(10,9,3,3,1)$ but want to allow more traffic than what has been observed so far. We can start with $\mathbf{v}$ larger than $\hat{\mathbf{v}}$ by any desired amount, say $\mathbf{v}=(10,10,5,5,2)$. Now $w(\hat{\mathbf{v}})=520$ while $w(\mathbf{v})=640$ and this $\mathbf{v}$ has a $23 \%$ slack built in.

\subsection{Cold Start}

With the adaptation mechanism defined earlier, it is possible to use TCS in a cold start mode, that is, with absolutely no information available about user traffic characteristics. We can start with $\mathbf{v}=(n, n, \ldots, n)$ allowing peak rate traffic or a smaller value provided it has sufficient slack built into it to account for unexpected situations. Now, as data is collected, $\mathbf{v}$ can be decreased progressively.

\subsection{Advanced TCS-Based Policing Mechanisms}

The number of variables and how they are updated can be easily changed to implement many advanced features.

Policing can be based on dual (or even multiple) TCS characterizations of user data. Thus, occasional data spikes can be characterized using a large window size, while the remaining data is characterized with a smaller window size. For illustrative purposes, let all cell counts in Figure 2 higher than 65 be considered as spikes in the data. There are four such counts in intervals 13, 24, 27 and 33. Thus, the primary TCS characterization will have a maximum threshold of 65 . The spikes will have to be admitted with a secondary TCS characterization. We can choose these as follows:

Primary TCS: $n_{1}=10, m_{1}=4, \mathbf{h}_{1}=(0,20,40,65), \mathbf{v}_{1}=(10,9,3,0)$.

Secondary TCS: $n_{2}=100, m_{2}=2, \mathbf{h}_{2}=(0,20), \mathbf{v}_{2}=(4,0)$.

Recall that for the data in Figure 2, we had measured $\mathbf{v}=(10,9,3,3,1)$ corresponding to $\mathbf{h}=(0,20,40,60,80)$. Our new characterizations correspond to these measurements.

The policing mechanism is changed to allow the sum of the two characterizations. The primary TCS allows up to 65 cells a maximum of 3 times in every 10 time intervals, etc. The secondary TCS allows an extra 20 cells to be admitted, over and above what is admitted by the primary TCS, a maximum of 4 times in 100 time intervals. The primary TCS allows 45.5 cells per time interval on the average. The secondary TCS allows 0.8 cells per time interval on the average. The two together 
allow 46.3 cells which is significantly less than the 52 cells allowed by $\mathbf{v}=(10,9,3,3,1)$ originally. Thus, using multiple characterizations reduces overdimensioning.

A variation of the dual scheme is to let the second TCS characterization denote the number of (marked) cells which will be admitted once the first TCS characterization is exceeded.

Another variation is to charge more for admitting under the second TCS characterization.

\section{CONCLUSIONS AND FUTURE WORK}

The types of user traffic characterization used currently have been shown to have several deficiencies. The peak rate based characterization is enforceable but leads to wasteful overdimensioning of the network. Long run statistical measures, such as the mean rate, standard deviation, etc., allow better allocation of network resources but cannot be effectively policed. We have developed a new thresholdbased characterization which is almost complete general and has immense flexibility together with the ability to be very specific. It considerably generalizes the moving window and peak rate allocation schemes. A policing mechanism based on this characterization has a reasonably simple implementation. Advanced services can be built on this basic policing mechanism. There are several parameters which can be tailored to each user's application. Easy-to-implement procedures are developed to obtain the remaining parameters directly from user data. The characterization can be used in a "cold start" mode when there is uncertainty about user behavior, and easily adapted as more data becomes available.

One aspect that clearly stands out is the generality of TCS. TCS has a large number of parameters but many of these are simply selections that the network and/or user must make. The parameters can be chosen to allow a wide variety of traffic patterns with the shape of allowable patterns specified much more precisely than the Leaky Bucket. The parameters can also allow any amount of excess traffic as desired. Thus, TCS promises to be a widely used scheme due to its unique combination of almost complete generality and immense flexibility together with the ability to be very specific.

We have developed TCS in its most general form. Simple ready-made versions of TCS with just a few thresholds will suffice for single users. More parameters will be used and more options allowed for services linking networks or involving a large amount of traffic. The "tailoring" of TCS is left for future work. 
TCS can be generalized by adding parameters which control the distance between successive threshold violations (see Section 4.2). The full development is left for future work.

Finally, the performance of TCS should be studied and compared with that of the Leaky Bucket, the Generalized Leaky Bucket (Johri (1997)), and other schemes, for a variety of settings. This study will be similar to the one in Rathgeb (1993). This is also left for future work.

\section{REFERENCES}

Butto, M., Cavallero, E. \& Tonietti, A., (1991), "Effectiveness of the Leaky Bucket policing Mechanism in ATM Networks," IEEE Journal on Sel. Areas in Comm. 9, 335-342.

Choudhury, G. L., Lucantoni, D. M. and Whitt, W., (1996), "Squeezing the Most Out of ATM," IEEE T. on Comm. 44, 203-217.

Doshi, B. T., (1994), "Deterministic Rule Based Traffic Descriptors for Broadband ISDN: Worst Case Behavior and Connection Acceptance Control," in The Fundamental Role of Teletraffic in the Evolution of Telecommunication Networks (Proceedings of ITC 14), edited by J. Labetoulle and J. W. Roberts, Vol. 1a, 591-600, Elsevier, New York.

Elwalid, A. \& Mitra, D., (1993), "Effective Bandwidth of General Markovian Traffic Sources and Admission Control of High Speed Networks," IEEE/ACM Transactions on Networking 1, 329-343.

Johri, P. K., (1995), "Estimating Cell Loss Rates in High Speed Networks with Leaky Bucket Controlled Sources," Int. Journal of Comm. Systems 8, 303-312.

Johri, P. K., (1997), "Apparatus and Method for a Generalized Leaky Bucket," United States Patent 5,625,622.

Rathgeb, E. P., (1992), "Policing of Realistic VBR Video Traffic - A Case Study," IFIP Trans.: C Communications Systems 4, 287-300.

Rathgeb, E. P., (1993), "Policing of Realistic VBR Video Traffic in an ATM Network," Int. J. of Digital and Analog Communication Systems 6, 213-226.

Turner, J. S., (1986), "New Directions in Communications (or which Way in the Information Age?)," Proc. Zurich Sem. Digit. Comm., Zurich, Switzerland, March 1986, 25-32.

Tutofor, K., (1990), "On Admission Control and Policing in an ATM-based Network," Proc. 7th ITC Seminar, New Jersey, October 1990, paper 5.4. 